\title{
Eye Tracking the Use of a Collapsible Facets Panel in a Search Interface
}

\author{
Max Kemman, Martijn Kleppe and Jim Maarseveen \\ Erasmus University Rotterdam, The Netherlands \\ kemman@eshcc.eur.nI
}

\begin{abstract}
Facets can provide an interesting functionality in digital libraries. However, while some research shows facets are important, other research found facets are only moderately used. Therefore, in this exploratory study we compare two search interfaces; one where the facets panel is always visible and one where the facets panel is hidden by default. Our main research question is "Is folding the facets panel in a digital library search interface beneficial to academic users?" By performing an eye tracking study with $\mathrm{N}=24$, we measured search efficiency, distribution of attention and user satisfaction. We found no significant differences in the eye tracking data nor in usability feedback and conclude that collapsing facets is neither beneficial nor detrimental.
\end{abstract}

Keywords. eye tracking, facets, information retrieval, usability, user studies, digital library, user behaviour, search user interface

\section{Introduction}

In the development of search interfaces for digital libraries, an interesting functionality is the availability of filters, or facets. Not only do facets help to refine the search results, they can also support the searcher by presenting an overview of the structure of the collection, as well as provide a transition between browsing and search strategies. In previous eye tracking studies, it was found that facets played an important role in the exploratory search process However, the authors stated that "it is possible that the visual layout influenced searchers - people click on what they see and what is most visible", leaving room for different results when implementing the facets differently [1]. In our previous research, academic researchers indicated that facets are not an important factor in their search process [2]. Facets could thus also be an unnecessary complexity in the interface. To research this, we created a simplified search interface with a collapsible facets panel. The panel is collapsed to a minimized form, until the user actively opens it. However, when hiding the facets, the opposite of "people click on what they see", namely "out of sight is out of mind [3] might occur. Therefore, in this exploratory study we compare two search interfaces; one where the facets panel is always visible and one where the facets panel is collapsible and thus hidden by default. Our main research question is "Is folding the facets panel in a digital library search interface beneficial to academic users?" In order to address this ques- 
tion, we performed an eye tracking study with academic users to evaluate the two search interfaces by analysing search efficiency, distribution of attention and user satisfaction. In doing so, we will test the hypothesis that collapsing the facets panel will be a detrimental user experience due to the "out of sight is out of mind" issue.

\section{Related work}

The benefit of using eye tracking for evaluating an interface are twofold [4]. First, eye tracking provides a more thorough insight in the interactions users have with the interface. Not only is data collected on user click behaviour, but also what users look at. As such, additional insight into the way attention is divided across the screen and the way information is processed can be acquired [5]. For example, a large number of eye movements across the screen can indicate a suboptimal layout, resulting in a less efficient search [6]. Other measures such as the amount of time spent on, or number of visits to, a certain area of the screen can provide information about the attentiongrabbing properties of such an area. This can serve a useful role in judging whether a certain aspect of the user interface is looked at, understood or is distracting the user. Second, it complements other qualitative data such as user feedback and think-aloud, as it provides a real-time insight in how users experience the interface. As such, eye tracking can be used to track down the source of usability issues found with traditional usability metrics [7].

\section{$3 \quad$ Method}

The interface we used to evaluate the collapsing of the facets panel is the PoliMedia system. This system is a search interface for the minutes in the Dutch parliament, linked to the media coverage of those debates [8]. Facets present in the interface are role (i.e. the role of the politician; parliamentary member or minister), politician (i.e. name), political party and year. We then created two versions of the system; 1) where the facets panel is visible and 2) where the facets panel is collapsible, see figure 1 . In the collapsible panel version, the facets panel collapsed up into a button called "Filters". The button was large enough and clearly marked so that users could easily recognize where the facets panel was, in order to address the "out of sight is out of mind" issue. We evaluated with a total of 24 participants, of which 11 participants received the visible version of the interface, while 13 received the collapsible version. Of these participants, 15 were male and 9 female. The average age was approximately 30, in the range of 22-45. Participants received a verbal introduction and completed five known item search (KIS) tasks and three exploratory search tasks, for which they recorded their answers on an answer sheet. Their eye tracking movements were recorded using the Mirametrix S2 Eye Tracker. To test the effect of collapsing the facets panel on the users' interaction with this panel, we divided the interface into five separate areas of interest (AOI's) based on their functionality: 1) search bar, 2) facets, 3) search results, 4) page-search (via ctrl+f command) and 5) other, containing remaining parts of the screen. 


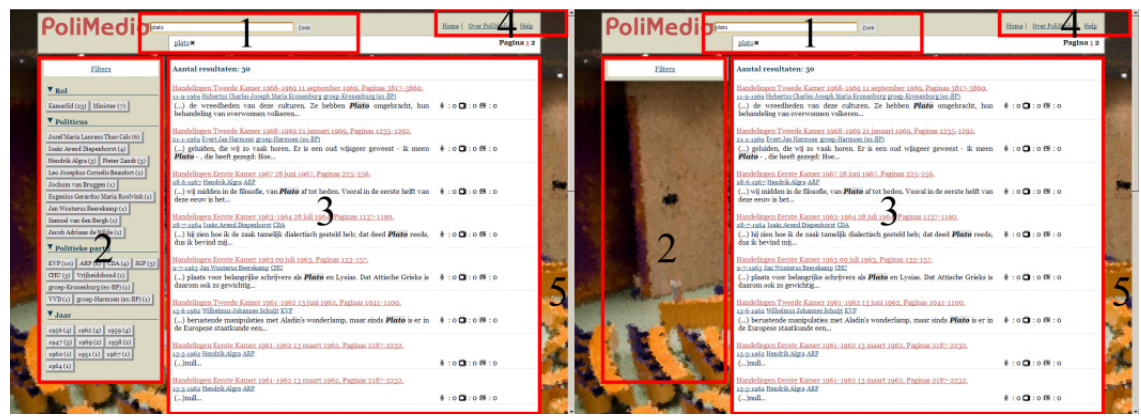

Fig. 1. Screenshots of the visible facets version (left) and the collapsible facets version (right) with Areas of Interest drawn on top of the screenshots

The eye tracking data was quantitatively analysed separately for both the KIS and the exploratory search sessions using the OGAMA ${ }^{1}$ statistical module and SPSS. After the recording errors were removed by manually checking video recordings of outliers we calculated the total number of fixations (NF) and total viewing duration (VD) overall and per AOI, to analyse respectively search efficiency and distribution of attention, as discussed in the related work. These measurements were analysed with four MANOVA's; for both the KIS and exploratory search sessions we used the viewing data (NF or VD) for each AOI as dependent variables and version (visible or collapsible facets) as the independent variable.

\section{$4 \quad$ Results $^{2}$}

We found that participants with the visible interface spent more total fixations (NF) and more total viewing duration (VD) on the KIS tasks than participants with the collapsible interface. However, for the exploratory tasks, this is the other way around. For the KIS tasks, participants with the visible version spent $18.2 \%$ of NF on the facets panel, and $20.2 \%$ of VD. Participants with the collapsible version spent $20.8 \%$ of $\mathrm{NF}$ and $22.3 \%$ of the VD on the facets panel. For the exploratory tasks, participants with the visible version spent $9.3 \%$ of the NF and $11.7 \%$ of VD on the facets panel. Participants with the collapsible version spent $8.5 \%$ of NF and $11.1 \%$ of VD on the facets panel. Comparing between the visible and collapsible facets versions using MANOVA with Pillai's trace, we found no significant differences for any of the metrics used. In other words, we found no significant differences in search efficiency, nor in distribution of attention. Moreover, we found no significant differences in users' satisfaction during the post-experiment discussion.

1 OpenGazeAndMouseAnalyzer: http://www.ogama.net/

2 All data minus the videos of the recordings are available open access through http://www.persistent-identifier.nl/?identifier=urn:nbn:nl:ui:13-lvo4-9k 


\section{Conclusion}

It appears that the facets were heavily used during searching in both versions of the search interface. We assume that the more moderate figures in the exploratory search can be explained by the users interacting much more with the speeches themselves, increasing the interaction with the search results AOI. Given the lack of significant differences between the two versions of the interface in users' eye tracking data nor in users' satisfaction between the two groups, we conclude that collapsing the facets panel did not introduce the usability issue "out of sight is out of mind", meaning it can provide a viable alternative to showing the facets at all times. We can thus reject our hypothesis. However, having the facets visible at all times did not introduce usability issues either. To answer our research question "Is folding the facets panel in a digital library search interface beneficial to academic users?" we conclude that folding the facets panel is neither necessarily beneficial nor detrimental.

Acknowledgements. We are grateful for the financial support from the EU FP7 project AXES ${ }^{3}$ - Access to Audiovisual Archives ICT-269980 and from the CLARIN-NL project PoliMedia ${ }^{4}$ - Interlinking multimedia for the analysis of media coverage of political debates.

\section{References}

1. Kules, B., Capra, R., Banta, M., Sierra, T.: What do exploratory searchers look at in a faceted search interface? Proceedings of the 2009 joint international conference on Digital libraries - JCDL '09. 313 (2009).

2. Kemman, M., Kleppe, M., Scagliola, S., Jongbloed, R., Beunders, H.: Mapping the use of digital sources amongst Humanities scholars in the Netherlands. Digital Humanities Congress. , Sheffield (2012).

3. Nielsen, J., Pernice, K.: Eyetracking Web Usability. New Riders (2010).

4. Balatsoukas, P.: An eye-tracking approach to the evaluation of digital libraries. In: Dobreva, M., O’Dwyer, A., and Feliciati, P. (eds.) User Studies for Digital Library Development. pp. 95-104. Facet Publishing (2012).

5. Poole, A., Ball, L.: Eye tracking in human-computer interaction and usability research: Current status and future prospects. In: Ghaoui, C. (ed.) Encyclopedia of Human-Computer Interaction. Idea Group, Inc., Pennsylvania (2005).

6. Goldberg, J.H., Kotval, X.P.: Computer interface evaluation using eye movements: methods and constructs. International Journal of Industrial Ergonomics. 24, 631-645 (1999).

7. Pretorius, M., Calitz, A., Greunen, D. van: The added value of eye tracking in the usability evaluation of a network management tool. SAICSIT 2005. 1-10 (2005).

8. Kemman, M., Kleppe, M.: PoliMedia - Improving Analyses of Radio, TV \& Newspaper Coverage of Political Debates. Theory and Practice of Digital Libraries. Springer, Valletta, Malta (2013).

3 http://www.axes-project.eu

4 http://www.polimedia.nl 